\title{
The Impact of Prior Assumptions on Bayesian Estimates of Inflation Parameters and the Expected Gravitational Waves Signal from Inflation
}

\author{
Wessel Valkenburg ${ }^{a *}$, Lawrence M. Krauss ${ }^{b \dagger}$, and Jan Hamann ${ }^{a \ddagger}$ \\ ${ }^{a} L_{A P T H} H^{\S}$ Université de Savoie and CNRS, 9 chemin de Bellevue, \\ BP110, F-74941 Annecy-le-Vieux Cedex, France and \\ ${ }^{b}$ School of Earth and Space Exploration and Department of Physics, \\ Arizona State University, PO Box 87104, Tempe, \\ Arizona 85287-1404, USA and Departments of Physics, \\ Case Western Reserve University, Cleveland, Ohio, USA
}

(Dated: 12 September 2008)

\begin{abstract}
There has been much recent discussion, and some confusion, regarding the use of existing observational data to estimate the likelihood that next-generation cosmic microwave background (CMB) polarization experiments might detect a nonzero tensor signal, possibly associated with inflation. We examine this issue in detail here in two different ways: (1) first we explore the effect of choice of different parameter priors on the estimation of the tensor-to-scalar ratio $r$ and other parameters describing inflation, and (2) we examine the Bayesian complexity in order to determine how effectively existing data can constrain inflationary parameters. We demonstrate that existing data are not strong enough to render full inflationary parameter estimates in a parametrization- and priorindependent way and that the predicted tensor signal is particularly sensitive to different priors. For parametrizations where the Bayesian complexity is comparable to the number of free parameters we find that a flat prior on the scale of inflation (which is to be distinguished from a flat prior on the tensor-to-scalar ratio) leads us to infer a larger, and in fact slightly nonzero tensor contribution at $68 \%$ confidence level. However, no detection is claimed. Our results demonstratethat all that is statistically relevant at the current time is the (slightly enhanced) upper bound on $r$, and we stress that the data remain consistent with $r=0$.

PACS numbers: $98.80 . \mathrm{Cq}$
\end{abstract}

\section{INTRODUCTION}

Shortly after its introduction [1, 2, 3, 4, 5, 6, inflation was found to produce a nearly flat Gaussian spectrum of adiabatic density perturbations that could have been the seeds of observed structure in the Universe $[7,8,9,10,11,12,13$. The simplest model of inflation is that of a slowly rolling scalar field [14, 15, 16, which naturally produces a close to flat primordial spectrum. While the available observations are remarkably consistent with such a spectrum, unfortunately one can obtain virtually any scalar spectrum by simply adjusting the shape of the inflaton potential at early times, and therefore present results are strongly suggestive, but not yet unimpeachable evidence that inflation actually occurred.

There are other more generic predictions of inflation that could be subject to testing, however. For example, a single rolling scalar field during inflation produces perturbations that are very close to Gaussian. A detection of significant primordial non-Gaussianity in the cosmic microwave background (CMB) could rule out simple slow-

\footnotetext{
*wessel.valkenburg@lapp.in2p3.fr

†krauss@asu.edu

¥jan.hamann@lapp.in2p3.fr

§Laboratoire de Physique Théorique d'Annecy-le-Vieux, UMR5108

^ current address
}

roll inflation [17]. A second possibility is the fact that inflation generally produces a spectrum of tensor perturbations, which could, among other effects, produce an observable $B$-mode polarization in the CMB [18, 19, albeit plagued by uncertainties [20, 21]. Note that tensor perturbations are not the only source of $B$-mode polarization 22, 23, 24, 25, and noninflationary transitions can also produce a similar background 26, 27. Nevertheless, observation of both the scalar spectrum and the tensor spectrum could at least test the predictions of slow-roll (SR) inflation, through the consistency relation

$$
n_{\mathrm{T}}=-r / 8, \quad \alpha_{\mathrm{T}}=n_{\mathrm{T}}\left[n_{\mathrm{T}}-n_{\mathrm{S}}+1\right], \quad \text { etc. },
$$

where $n_{\mathrm{T}}$ is the tilt of the tensor spectrum, $r$ is the ratio of the amplitudes of the tensor and scalar spectra, $\alpha_{\mathrm{T}}$ is the running of the tensor spectrum and $n_{\mathrm{S}}$ is the tilt of the scalar spectrum. A tensor spectrum has not been detected so far, and many future experiments have been proposed to search for a gravitational waves signal from inflation [28, 29, 30, 31, 32, 33, 34, 35, 36, 37.

With only observations that constrain the scalar spectrum, one might hope to gain some information on the inflaton potential [38, 39, 40. However the plethora of different models of inflation make such a task difficult. Nevertheless, obtaining any information one can on the potential using the observed scalar perturbations could give information about the possibility of observing tensor perturbations. In Ref. [41] the following relation between the change in value of the scalar field $\phi$ and the tensor-to-scalar ratio $r$, holding deep inside the slow-roll 
approximation, was pointed out,

$$
\frac{1}{m_{\mathrm{P}}} \frac{\Delta \phi}{\Delta N} \simeq \sqrt{\frac{r}{64 \pi}},
$$

where $N$ is the number of $e$-folds the Universe grows $d u r$ ing the change $\Delta \phi$ of the scalar field. That is, when focusing on only a small part of the potential, and not necessarily on the whole duration of inflation, $\Delta N$ can correspond to a number much smaller than the total number of $e$-folds of inflation, $N \sim 60-70$. Hence, relation (2) relates the flatness of the potential to the relative amplitude of tensor perturbations. Throughout this work we use $G m_{\mathrm{P}}^{2}=\hbar=c=1$.

As the only current probe of the mechanism of inflation is the observed spectrum of density perturbations in the Universe, Refs. [42, 43, 44, 45, 46, 47] concentrated on reconstructing the inflaton potential only in the observational range. It was found in Ref. [46] that in the observational range naturally $\Delta \phi<m_{\mathrm{P}}$ and $\Delta N \sim 22$. This bound on $\Delta N$ comes from the condition that the smallest observable modes actually freeze in [47. The bound on $\Delta \phi$ can then be understood from Eq. (2) as the data prefer models with $r$ smaller than at most 0.4 (depending on the data used).

The reconstruction of the inflation potential gave a weak upper limit on $r$, fully consistent with $r=0$. More recently, however, at least one group has claimed that recent data imply a nonzero lower limit on $r$ [4].

Obviously it is important to clarify this situation, especially when the results would have such great significance, and when a dedicated satellite mission to probe for primordial $B$ modes associated with a nonzero tensor signal, is being considered.

In cases such as this, it is useful to take a Bayesian approach and to consider how effective the data really are at constraining parameters. Thus, one must consider not merely a posteriori probability estimations, but also the effect of prior assumptions (see [4] for some discussion of this issue). If the results depend crucially on the latter, then the parameter estimates one derives from the data must be taken with a grain of salt.

The purpose of this paper is to explicitly explore precisely this question at the current time, in order to help solidify expectations for future measurements of this important and fundamental quantity arising from inflation. Specifically we first explore to what extent the priors one assumes in the analysis affect the expected value of $r$.

One might argue that with little knowledge of the relevant physics, it is perhaps pointless to argue strongly on behalf of one set of priors or another at this point. It does make sense, however, to examine how robust the conclusions one draws are, under different prior assumptions. (See also work to appear by Vaudrevange and colleagues [50, 51.) This work focuses on the effect of taking a flat prior on the Hubble factor during inflation, and its derivatives with respect to the scalar field value $\phi$. We will show that a change of parametrization, but not of physical model, in this case can lead to significantly different bounds on parameters, some of which may mildly hint at a larger value of $r$ as well.

In this regard we note that in Ref. 48, a lower bound on the tensor-to-scalar ratio has been found which one might be tempted to ascribe to a choice of prior. An important difference between their result and ours however is that their lower bound on the tensor-to-scalar ratio is caused by a theoretical prior: the models they allow can only be consistent with today's observed scalar amplitude and tilt if the tensor-to-scalar ratio is significant. In the present work however, the prior on allowed models is as broad as possible, a priori not ruling out any combination of inflationary parameters.

Next, in order to explore the general significance of any derived lower bound on $r$ based on a choice of priors, we examine the Bayesian complexity parameter associated with the current data. This gives a very useful tool to explore how many free parameters the data can usefully constrain. As we demonstrate, for many inflationary parametrizations, the data are currently simply not powerful enough to add information beyond the prior, for all the parameters, explaining the prior-dependence of estimates of $r$ that we have found. Thus, we argue that existing data at best provide a rough upper bound on $r$, rather than providing a robust estimate of its posterior probability distribution.

In Sec. II we discuss the relation between different flat priors, and explain how to translate posterior probability densities from one prior to another. In Sec. III we apply a flat prior on the value of the Hubble parameter and its derivatives during inflation, fit it to the data, and discuss the results for both prior dependence and Bayesian complexity. We conclude in Sec. IV.

\section{PRIORS AND POSTERIORS}

When faced with the problem of estimating parameters from data, Bayesian inference enjoys a great popularity among cosmologists (see 52 for a recent review). An essential ingredient of any Bayesian inference is the prior distribution, which encodes our knowledge about these parameters before any data are taken. With a suitable basis of parameter space $\left\{x_{i}\right\}$ chosen, it is often tacitly assumed that the prior is flat - signifying our lack of information about this parameter in the absence of data. In other words, the prior probability of an interval $\Delta x_{i}$ to contain the true value of the $x_{i}$ is taken to be constant over the entire domain of definition of parameter space.

However, while in some problems there is a naturally preferred basis of parameter space, this need not always be the case, and an alternative, equally well motivated parametrization $\left\{y_{i}\right\}$ may exist. It is straightforward to show that generally, a prior in basis $\left\{x_{i}\right\}$ does not correspond to the same prior in basis $\left\{y_{i}\right\}$. Labeling a prior $\mathrm{A}$ on $\left\{x_{i}\right\}$ by $\pi_{x}^{(A)}$, the corresponding prior on $\left\{y_{i}\right\}$ is given 
by

$$
\begin{aligned}
\int \pi_{x}^{(A)} d^{n} x & =1 \\
& =\int \pi_{x}^{(A)}\left|\frac{d x_{i}}{d y_{j}}\right| d^{n} y \\
& \equiv \frac{1}{V_{y}} \int \pi_{y}^{(A)} d^{n} y, \\
\pi_{y}^{(A)}(\vec{y}) & \propto \pi_{x}^{(A)}(\vec{x}(\vec{y}))\left|\frac{d x_{i}}{d y_{j}}\right|,
\end{aligned}
$$

where $V_{y}=\int d^{n} y$. Hence a flat, noninformative prior in one basis does not necessarily equal a noninformative prior in another, making the choice of basis equivalent to the choice of prior, and by consequence, extending its influence to the posterior and the inferred parameter constraints, unless the data become informative enough. This problem was identified in [53, 54, 55] in the context of isocurvature models; here we will argue that inflationary parameters, including estimates of the tensorto-scalar ratio, can also be affected.

\section{A. Importance sampling}

If from earlier analyses one knows that the bounds on parameters in set $\left\{x_{i}\right\}$ have Gaussian-like shapes, and the sets $\left\{x_{i}\right\}$ and $\left\{y_{i}\right\}$ are nonlinearly related, one can expect that correlations between parameters in set $\left\{y_{i}\right\}$ are of nontrivial shape. In that case a Metropolis-Hastings algorithm, which is what we will use later on, will have difficulty exploring parameter space properly within an acceptable amount of time. A solution to this problem is importance sampling, which is the act of picking points according to one posterior distribution, but transforming the chance of accepting the point to another posterior distribution. In this way the algorithm walks through parameter space according to directions in the 'easierto-explore' $\left\{x_{i}\right\}$-space, but performing the statistics as if working in $\left\{y_{i}\right\}$-space. The resulting chains will be distributed according to the prior chosen in $\left\{y_{i}\right\}$-space. Let $A$ denote statistics with a flat prior on $\left\{x_{i}\right\}$, and let $B$ denote statistics with a flat prior on $\left\{y_{i}\right\}$. In the Metropolis-Hastings algorithm, the chance of accepting a proposed step is directly related to the ratio of its posterior and the posterior of the previous point. Hence a constant multiplicative factor in the posterior is irrelevant, and we can neglect the volume term in Eq. (4). By consequence, any constant prior corresponds to a flat prior, such that the conversion to be done is

$$
\begin{aligned}
\pi_{y}^{(B)}(\vec{y}) & =\left|\frac{d y_{i}}{d x_{j}}\right| \pi_{y}^{(A)}(\vec{y})=\text { constant, } \\
\mathcal{P}^{(B)}(\vec{y}(\vec{x})) & =\left|\frac{d y_{i}}{d x_{j}}\right| \mathcal{P}^{(A)}(\vec{x}) .
\end{aligned}
$$

There are two distinct places in the analysis in which the correction for the prior can be applied. One option, which we shall refer to as post-sampling, is to take the converged chains of an analysis performed under prior $(A)$, and multiply the weight of each point in the chain by the Jacobian as in Eq. (6).

The advantage is that one can post-process readily available chains to present a new prior, which takes practically no time. A possible drawback is that the chains, that converged for an analysis under prior A, may have too few (or no) points in the regions of parameter space important under prior B.

The second option is explicit importance sampling of the second distribution, in which one, during the MonteCarlo process, transforms the posterior of a point to reflect the correct prior, by applying Eq. (6) before the decision about acceptance of the point is taken. The advantage is that the convergence statistics will now be performed for the correct probability density, hence important regions will have enough points in the chains. A drawback is that the analysis has to be performed from scratch, which can be time consuming.

\section{B. Cosmological parameters}

When constraining the parameters of $\Lambda \mathrm{CDM}$ cosmologies, it is a popula choice to take flat priors on $\left\{\Omega_{\mathrm{c}} h^{2}, \Omega_{\mathrm{b}} h^{2}, \tau, \theta\right\}$ (the dark matter density, the baryon density, the optical depth to reionization, and the ratio of sound horizon to angular diameter distance at decoupling, respectively) and for the primordial power spectrum a flat prior on either $\left\{\ln A_{\mathrm{S}}, n_{\mathrm{S}}, \alpha_{\mathrm{S}}\right\}$ (the amplitude, tilt, and running of the spectrum) or $\left\{\ln A_{\mathrm{S}}, \epsilon_{i}\right\}$, with $\left\{\epsilon_{i}\right\}$ some basis of slow-roll (SR) parameters. In the SR-basis of Hubble-flow parameters, the dynamics of inflation are hidden in these parameters by

$$
\begin{aligned}
A_{\mathrm{S}} & =\frac{4 H_{*}^{4}}{H_{*}^{\prime 2} m_{\mathrm{P}}^{4}}, \\
\epsilon & =\frac{m_{\mathrm{P}}^{2}}{4 \pi}\left(\frac{H_{*}^{\prime}}{H_{*}}\right)^{2}, \\
H_{*} & =\frac{m_{\mathrm{P}}^{2}}{2} \sqrt{\pi A_{\mathrm{S}} \epsilon} .
\end{aligned}
$$

where $H_{\mathrm{Inf}}=H_{*}$, '*' denotes evaluation at the pivot scale, and ' denotes derivation with respect to the field value of the inflaton. This means that in all these analyses the posterior distribution of the derived parameter $H_{\text {Inf }}$ is obtained with a nonflat prior.

In Fig. 1 we show the Jacobian $\left|\frac{d x_{i}}{d y_{j}}\right|=\frac{4 H_{*}^{\prime 2} m_{\mathrm{P}}^{6}}{H_{*}^{6}}$ relating 


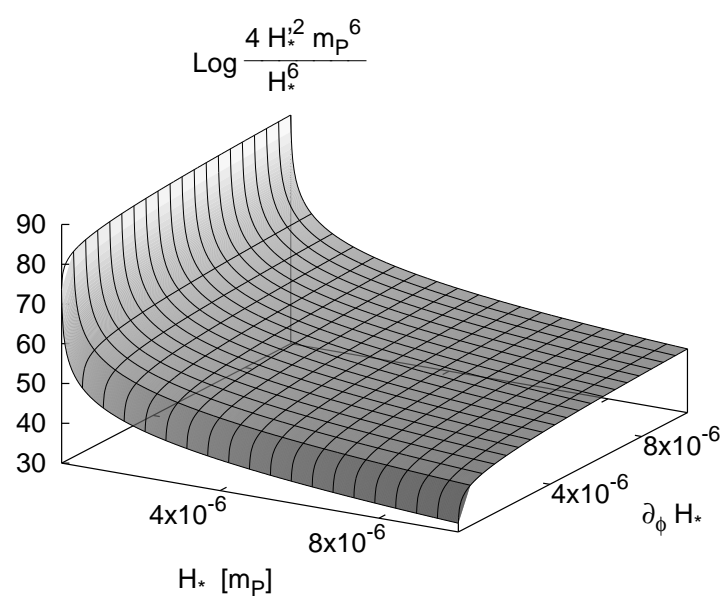

FIG. 1: The Jacobian $\left|\frac{d x_{i}}{d y_{j}}\right|$ for the coordinate transformation from set $\left\{x_{i}\right\}$ to set $\left\{y_{i}\right\}$. It is clear that a flat prior on set $\left\{x_{i}\right\}$ strongly favors small values for $H_{*}$ compared to a flat prior on set $\left\{y_{i}\right\}$, as $\pi_{y}^{(A)} \sim \pi_{x}^{(A)}\left|\frac{d x_{i}}{d y_{j}}\right|$.

the coordinate transformation between sets

$$
\begin{aligned}
& \left\{x_{i}\right\} \equiv \\
& \left\{\ln \frac{4 H_{*}^{4}}{H_{*}^{\prime 2} m_{\mathrm{P}}^{4}},\left(\frac{H_{*}^{\prime}}{H_{*}}\right)^{2} m_{\mathrm{P}}^{2}, \frac{H_{*}^{\prime \prime}}{H_{*}} m_{\mathrm{P}}^{2}, \frac{H_{*}^{\prime \prime \prime} H_{*}^{\prime}}{H_{*}^{2}} m_{\mathrm{P}}^{4}\right\} \\
& \left\{y_{i}\right\} \equiv\left\{\frac{H_{*}}{m_{\mathrm{P}}}, H_{*}^{\prime}, H_{*}^{\prime \prime} m_{\mathrm{P}}, H_{*}^{\prime \prime \prime} m_{\mathrm{P}}^{2}\right\}
\end{aligned}
$$

corresponding up to a constant to the ratio $\pi_{y}^{(A)} / \pi_{x}^{(A)}$. A flat prior on set $\left\{y_{i}\right\}$ (prior B) favors high values of $H_{*}$ when compared to a flat prior on set $\left\{x_{i}\right\}$ (prior A).

Once the data come into play, the amplitude $A_{\mathrm{S}}$ will essentially be fixed. Since $A_{\mathrm{S}} \propto H_{*}^{2} / \epsilon$, higher values of $H_{*}$ will need to be offset by higher values of $\epsilon$. In the slow-roll regime, $\epsilon$ is related to the tensor-to-scalar ratio $r$ by

$$
r \simeq 16 \epsilon=\frac{4 m_{\mathrm{P}}^{2}}{\pi}\left(\frac{H^{\prime}}{H}\right)^{2}
$$

and hence we can expect prior B to prefer a larger tensor contribution, compared to prior A. Equation (12) also shows that a flat prior on $\epsilon$ roughly corresponds to a flat prior on $r$.

\section{FLAT PRIOR ON $H_{\text {Inf }}$}

In order to probe the scale of inflation, we numerically integrate the perturbation equations of the inflaton in a background described by a Taylor-expansion of $H(\phi)$, as discussed in 45, 46, and constrain the free parameters using temperature and polarization data from
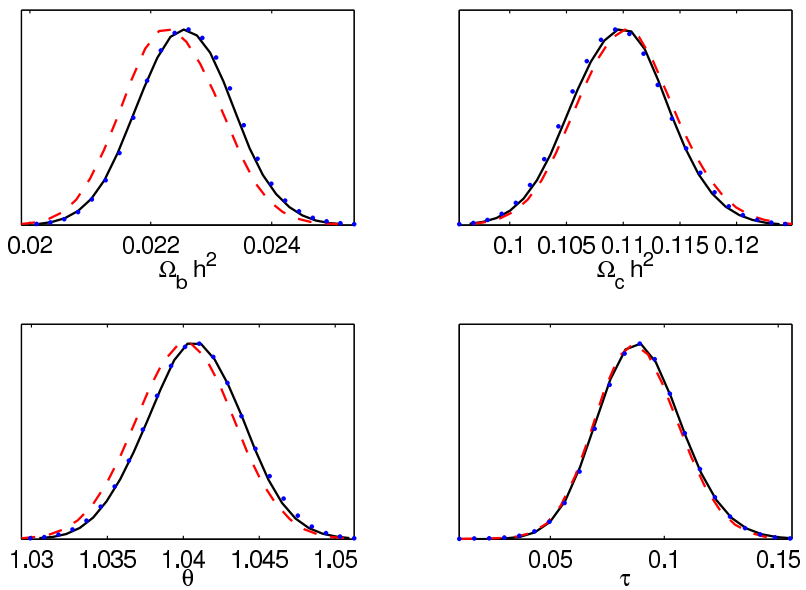

FIG. 2: The marginalized posterior distributions of cold dark matter density $\left(\Omega_{\mathrm{c}} h^{2}\right)$, the baryon density $\left(\Omega_{\mathrm{b}} h^{2}\right)$, the ratio of sound horizon to angular diameter distance at decoupling $(\theta)$ and the optical depth to reionization $(\tau)$, under prior A (red dashed line), post-sampled from prior A to prior B (blue dotted line) and under prior B (black solid line). The postsampled distributions are hardly visible as they practically completely agree with the importance sampled distributions.

the five year data release of the Wilkinson Microwave Anisotropy Probe (WMAP) satellite (WMAP5) [56], as well as the power spectrum of luminous red galaxies from the Sloan Digital Sky Survey (SDSS-LRG) [57]. The parameter estimation is done using the MetropolisHastings algorithm, employing a modified version of the publicly available code CosmoMC [58] together with our own module for inflationary perturbations (which is available for download at http://wwwlapp.in2p3. $\mathrm{fr} /$ valkenbu/inflationH/). The parameters describing the model are either $\left\{\Omega_{\mathrm{c}} h^{2}, \Omega_{\mathrm{b}} h^{2}, \tau, \theta\right\}+\left\{x_{i}\right\}$ or $\left\{\Omega_{\mathrm{c}} h^{2}, \Omega_{\mathrm{b}} h^{2}, \tau, \theta\right\}+\left\{y_{i}\right\}$. We include the calculation of tensor perturbations.

As a consequence of this exact numerical treatment of perturbations, we automatically impose a consistent inflationary prior (in the following this is referred to as "inflationary consistency"). By numerically integrating the perturbation equations until the actual freezein of all modes, this method requires inflation to occur over the observable range, which constrains parameters more strongly than a naive application of the SRapproximation. As pointed out in Ref. [47], a naive implementation of the SR-approximation allows for inconsistent models, for which small scale modes actually do not freeze in, even though the approximation provides a spectrum. We make no prior assumption on the total length of inflation other than the length needed to produce the observed power spectrum of perturbations. That is, we remain conservative about the mechanism of inflation during the unobserved epoch, between horizon exit of the smallest observable modes and the end of inflation.

As a consistency check we performed both described 

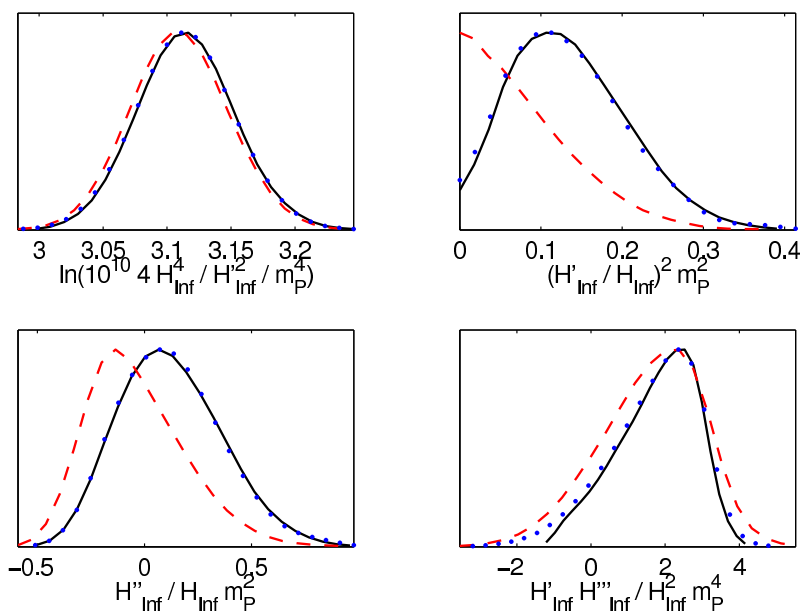

FIG. 3: The marginalized posterior distributions of the parameters describing the evolution of the Universe during inflation, for the same analyses as in Fig. 2. Again the postsampled distribution (blue dotted line) is hardly visible due to its good agreement with the importance sampled distribution (black solid line). The main change under the transformation of priors is seen in the posterior of $\left(\frac{H^{\prime}}{H}\right)^{2} m_{\mathrm{P}}^{2}$, in agreement with the prediction in Fig. 1
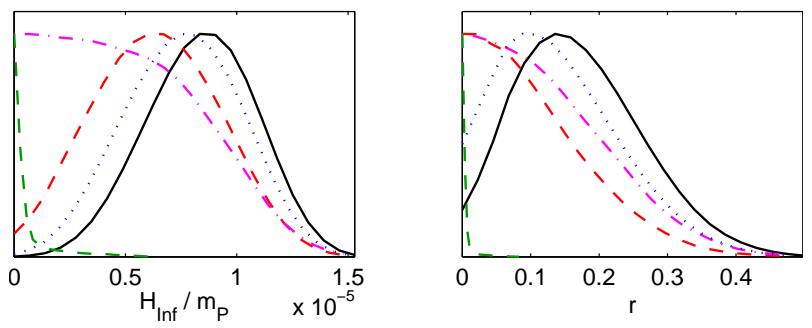

FIG. 4: The marginalized posterior distributions for the scale of inflation, $H_{\text {Inf }}$, and the scalar-to-tensor ratio, $r$, under prior A (red dashed line), under prior B (black solid lide), post-sampled to a Jeffreys prior on $H_{*}$ (thin blue dotted line) and under a flat prior on $\left\{A_{s}, \ln \epsilon, \frac{H_{*}^{\prime \prime}}{H_{*}} m_{\mathrm{P}}^{2}, \frac{H_{*}^{\prime \prime \prime} H_{*}^{\prime}}{H_{*}^{2}} m_{\mathrm{P}}^{4}\right\}$ (green dashed, close to zero for both figures). Prior B corresponds to a flat prior on $H_{\mathrm{Inf}}$, whereas prior A roughly corresponds to a flat prior on $r$, as explained in the text. Prior B pushes both $H_{\text {Inf }}$ and $r$ up in value. Also shown is the mean likelihood over each $(8-1)$-dimensional parameter space for all values of $H$ and $r$ (dashed-dotted, magenta).

methods, post-sampling and importance sampling. In Fig. 2 we show the one dimensional marginalized posterior distributions of the four cosmological parameters describing the physics after inflation, comparing the analyses with prior A, the post-sampled chains from prior A to prior B, and the chains with prior B (importance sampled). The post-sampled and importance sampled analyses completely agree, which shows that the chains that converged under prior A have enough samples in the typical set of the posterior distribution under prior B. As should be expected, the four parameters shown in Fig. 2 are not affected by the change in prior. In Fig. 3 we show
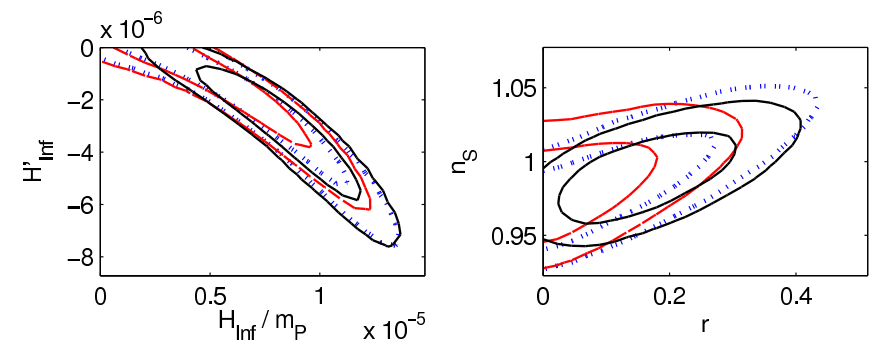

FIG. 5: Two dimensional marginalized posterior distributions for two illustrative cases, comparing prior A (red dashed line), prior B (black solid) and a noninflationary analysis, probing the four cosmological parameters plus the set $\left\{\ln A_{\mathrm{S}}, n_{\mathrm{S}}, \alpha_{\mathrm{S}}, r\right\}$ describing the primordial spectrum (blue dotted line). All inner contours correspond to $68 \%$ CL bounds, all outer contours correspond to 95\% CL bounds. Left: the curved correlation shape between $\partial_{\phi} H_{\text {Inf }}$ and $H_{\text {Inf }}$ illustrates the need for importance sampling when taking a flat prior on these parameters. Right: both $r$ and $n_{\mathrm{S}}$ are pushed up by prior B.

the posterior distributions of parameters describing the inflationary evolution. The main change is in the posterior of the parameter $\left(\frac{H^{\prime}}{H}\right)^{2} m_{\mathrm{P}}^{2}$, which has a higher preferred value under prior $\mathrm{B}$ than under prior $\mathrm{A}$. This result is in agreement with the expected effect, illustrated in Fig. 1.

The scale of inflation and the tensor-to-scalar ratio are shown in Fig. 4. Both parameters, which are related, have a higher preferred value under prior B.

For illustration, in Fig. 4, we also show a post-sampled distribution with a flat prior on $\left\{\ln H_{*}, H_{*}^{\prime}, H_{*}^{\prime \prime}, H_{*}^{\prime \prime \prime}\right\}$. Also this prior gives a higher value for $H_{*}$ and $r$ than prior A does. Note that under both prior A and prior $\mathrm{B}$, we find a lower $r$ than the combined analysis of the WMAP three year data and SDSS-LRG in Ref. [57, which is due to our inflationary prior: integrating the modes until actual freeze-in, means demanding inflation for about $22 e$-folds, which forces the inflaton potential to be relatively smooth. The smoothness of the potential pushes $\epsilon$ down and thereby also $r$. Likewise the scalar tilt $n_{\mathrm{S}}$ is pushed toward unity, as is shown in Fig. 5 where the two dimensional parameter correlations are shown for two illustrative cases, $H_{\text {Inf }}$ versus $\partial_{\phi} H$, and $r$ versus $n_{\mathrm{S}}$. The former illustrates the nonlinear correlation between the parameters $H_{\text {Inf }}$ and $\partial_{\phi} H$ in the data. The curved shape of the posterior probability contour indicates that it would take a Metropolis-Hastings sampler a long time to random-walk from one lobe to another if steps are only to be taken in either horizontal or vertical direction, or a linear combination of both, in the plane of this plot. By using importance sampling, steps are taken in correlated directions, significantly speeding up the process. The latter shows both the effect of the inflationary prior, present in both analyses, and the effect of going from prior A to prior B. In both analyses the value of $\epsilon$ is relatively close to zero, however it is larger under prior B. 
It is interesting to note that we also find an apparent lower bound on the scale of inflation, even for a flat prior on $H_{*}$. In fact, this phenomenon is related to our choice of prior on $\epsilon$ (or $H_{*}^{\prime}$, under prior B). Let us illustrate the effect in the example of prior $\mathrm{A}$. The dislike of the data for a large tensor contribution leads to an upper bound on $\epsilon$ due to Eq. 12. In order to reproduce the observed amplitude of fluctuations, $A_{\mathrm{S}} \sim H_{*}^{2} / \epsilon$ implies also an upper bound on $H_{*}$. However, as a consequence of the flat prior on $\epsilon$, extremely small values of $\epsilon$, while certainly allowed by the data, are assigned an exponentially suppressed probability, with a preference for $\epsilon$ of the order of magnitude of its upper bound. Since $A_{\mathrm{S}} \sim H_{*}^{2} / \epsilon$, we also have a suppression of small values of $H_{*}$, with a peak slightly below the upper bound. If we instead take the prior to be flat on the logarithm of $\epsilon$ (i.e., a Jeffreys prior on $\epsilon$ ), we do not see such a suppression. However, the results for the Jeffreys prior on $\epsilon$ must be interpreted with care as they are highly dependent on the lower bound. For numerical reasons we took a lower bound of $\ln \epsilon>-57$. Had we taken an even smaller lower bound, the lines would be even closer to zero. A similar result can be anticipated for a flat prior on $\ln H_{*}^{\prime}$ in the $\left\{y_{i}\right\}$ parametrization. That this dependence on the lower bound does not occur under the Jeffreys prior on $H$ but a flat prior on $H^{\prime}$ is explained by the same reasoning as the apparent lower bound on $H$.

In addition to the posteriors, Fig. 4 shows the mean likelihood over each $(8-1)$-dimensional parameter space for all values of $H$ and $r$. This is a prior-independent quantity with no probabilistic information (i.e., it is not a probability density). It serves as an approximation for the profile likelihood. The profile likelihood is the best fit that can be achieved given a certain parameter value. The discrepancy between the mean likelihood and the various posteriors indicates that the various lower bounds in the posteriors are results of either volume effects in the process of marginalization, the choice of prior, or a combination of both. The mean likelihood shows that a good fit can even be achieved for very small values of $r$ and $H_{\text {Inf }}$. In fact the best fit we found lies at $r=4 \times 10^{-2}$ and $H_{\text {Inf }}=4 \times 10^{-6} m_{\mathrm{P}}$. This certainly does not coincide with the peaks of the posteriors found for priors $\mathrm{A}$ and B.

In Fig. 5 we also compare derived parameters from the different analyses with the bounds obtained on these parameters when using no inflationary prior and simply fitting a primordial power spectrum,

$$
P(k)=A_{\mathrm{S}}\left(\frac{k}{k_{*}}\right)^{n_{\mathrm{S}}-1+\frac{1}{2} \alpha_{\mathrm{S}} \ln \frac{k}{k_{*}}+\ldots},
$$

and a consistent tensor spectrum, described by $r$ and consistency relations between the tensor spectral tilt $\left(n_{\mathrm{T}}\right)$ and the scalar parameters, to the same data. Calculating $\left\{H_{\text {Inf }}, H_{\text {Inf }}^{\prime}\right\}$ from $\left\{A_{\mathrm{S}}, n_{\mathrm{S}}, \alpha_{\mathrm{S}}, r\right\}$ is done using the relations given in Ref. [45]. The curved shape of the correlation between $H_{\mathrm{Inf}}^{\prime}$ and $H_{\mathrm{Inf}}$ reflects the need for importance sampling. The $95 \%$ confidence level (CL) con-

\begin{tabular}{l|l|l|l|l} 
& Prior A & Prior B & $\left\{A_{\mathrm{S}}, n_{\mathrm{S}}, \alpha_{\mathrm{S}}, r\right\}$ & $\begin{array}{l}\left\{A_{s}, \ln \epsilon, \frac{H_{*}^{\prime \prime}}{H_{*}} m_{\mathrm{P}}^{2},\right. \\
\left.\frac{H_{*}^{\prime \prime \prime} H_{*}^{\prime}}{H_{*}^{2}} m_{\mathrm{P}}^{4}\right\}\end{array}$ \\
\hline$C_{b}$ & $6.98 \pm 0.03$ & $7.80 \pm 0.03$ & $7.76 \pm 0.06$ & $6.25 \pm 0.8$
\end{tabular}

TABLE I: Bayesian complexity for different choices of prior. This number should be compared to the number of free parameters, which is eight for all models considered here.

tours under prior A correspond to the 95\% CL contours from the spectral fit for small values of $H_{\text {Inf }}^{\prime}$, whereas the 95\% CL contours under prior B correspond to the spectral fit for large values of $H_{\text {Inf }}^{\prime}$. An important conclusion to draw here is that both priors $\mathrm{A}$ and $\mathrm{B}$ allow most of parameter space that is allowed by the spectral fit, which has no inflationary prior.

In the $n_{\mathrm{S}}-r$-plane, prior A clearly pushes $r$ down with respect to merely performing a spectral fit because of the demand that inflation lasts long enough to produce the full observed spectrum under a flat prior on virtually the same parameters, whereas prior B pushes $r$ up, in spite of the same condition on the duration of inflation.

In the absence of clearly favored theoretical models, the beauty of various priors is, alas, largely in the eye of the beholder. Nevertheless we emphasize that both priors A and B do not exceed the spectral limits but do probe practically the whole range allowed without the requirement of persistent inflation. More interestingly, the $68 \%$ CL contour for prior B actually yields a nonzero lower bound for $r$. Marginalized over all parameters, the posterior of $r$ gives, at $68 \% \mathrm{CL}, 0.061<r<0.243$, however at $95 \%$ CL $r$ is still consistent with zero. While this may hint at a nonzero amplitude of tensor modes, our analysis underscores the prior model dependence of this result and thus we do not put much stake in it here. Nevertheless, it does suggest that future polarization searches for tensor modes may have a better chance of detection than otherwise suggested.

\section{Bayesian Complexity}

When selecting models and priors, a quantity that can distinguish between models is the Bayesian evidence, which rewards both the predictivity and the conciseness of a model, and gives preference to the model with the best balance between the two characteristics. When the Bayesian evidence cannot distinguish between two models, a secondary quantity to make the comparison is the Bayesian complexity [59, 60],

$$
\mathcal{C}_{\mathrm{b}} \equiv \overline{\chi^{2}}-\chi^{2}(\hat{\theta})
$$

where the effective $\chi^{2}$ is defined as $-2 \ln \mathcal{L}$, with the likelihood $\mathcal{L}$, and $\hat{\theta}$ denotes the best fit point, and the overline denotes the mean over the posterior. The Bayesian complexity measures the information gain when going from the prior to the posterior, and can be interpreted 
as a measure of the number of parameters the data can constrain in a model, or conversely the number of parameters a model effectively needs to fit the data. Along the same lines, if the Bayesian complexity is smaller than the actual number of free parameters of a model, this could be taken as a sign that the model contains "unnecessary" degrees of freedom, i.e., parameters on which we do not gain information from the data.

Note that the Bayesian complexity itself contains no information about the goodness of fit of a model, or the evidence of one model over another, but gives an additional measure on the number of parameters in a model that is justified by the data. Without an evidence calculation, the complexity can still be useful for telling whether parameters are mostly bounded by either the data or the prior. In this work we are interested in the question which priors (with the same underlying model) constrain parameters beyond the constraining power of the data, and which priors allow the data to give information on parameters.

The Bayesian evidence cannot be reliably calculated from the Markov-chain Monte Carlo (MCMC) chains obtained doing the parameter estimation, as these chains have a lack of information on the tails of the parameter distributions. An elaborate analysis would be necessary, e.g. using nested sampling [61. The Bayesian complexity, however, can be readily calculated from the chains. In Table I, we show the Bayesian complexity for the same model under the different priors.

For prior A we find a complexity of $6.98 \pm 0.03$, for eight free parameters. This indicates that the data do not give any information on one of the free parameters. Most likely this is due to $\frac{H_{*}^{\prime \prime \prime} H_{*}^{\prime}}{H_{*}^{2}} m_{\mathrm{P}}^{4}$ which is more tightly constrained by imposing inflationary consistency than by the data, as explained in Ref. [47. Compared to prior $\mathrm{A}$, prior $\mathrm{B}$ has more volume in regions constrained by the data, increasing the amount of information gained and pushing up the complexity to $7.80 \pm 0.03$. Compared to that, in the $\left\{A_{s}, \ln \epsilon, \frac{H_{*}^{\prime \prime}}{H_{*}} m_{\mathrm{P}}^{2}, \frac{H_{*}^{\prime \prime \prime} H_{*}^{\prime}}{H_{*}^{2}} m_{\mathrm{P}}^{4}\right\}$ basis the opposite happens, as $\epsilon$ is pushed much closer to zero, such that the data give no new information on this parameter, decreasing the complexity. For the "phenomenological" parameter set $\left\{A_{\mathrm{S}}, n_{\mathrm{S}}, \alpha_{\mathrm{S}}, r\right\}$ with flat priors, no inflationary consistency is imposed. Therefore, in this basis, $\alpha_{\mathrm{S}}$ has no theoretical prior constraints and can be constrained by the data.

An increase in the complexities under prior B and the phenomenological prior compared to prior A should not be taken to mean that these prior choices are superior. Indeed, in all cases the complexity value is less than 8 , which is the number of inflationary parameters under consideration. Rather, we take this as an indication that the data is highly sensitive to the choice of parametrization of inflationary models, in particular the choice of prior distribution for $r$, and hence the posterior probability densities reflect far more the choice of volume of prior parameter space than the impact of the data.

\section{CONCLUSION}

Our paper makes explicit an important and well-known fact regarding the effort to constrain cosmological parameters: the importance of prior assumptions in the analysis must not be neglected. We have demonstrated in a variety of ways that this situation is relevant to the current issue of a possible nonzero value of $r$ and expectations for future CMB missions. In the absence of clear theoretical direction, it is important therefore to consider the divergence of results obtained by presumably equally well motivated priors. We have demonstrated here how to relate flat priors on different parametrizations of the same physics, and applied a change of parametrization to the reconstruction of the inflaton potential, choosing a flat prior on the parameters that may be better motivated by the physics of inflation, as opposed to parameters describing the observable quantities. The main change, seen in Figs. 4 and 5 , is an increase of the preferred value for the tensor-to-scalar ratio $r$, moving from $0<r<0.18$ to $0.061<r<0.243$ at $68 \% \mathrm{CL}$ We stress once again that this new preferred range does not imply that the data now prefer a nonzero value of $r$, since at 95\% CL $r$ is consistent with zero under all used priors. The fact that for certain choices of parametrization the complexity is less than the number of free parameters, eight, indicates that the data is currently insufficient to fully constrain the models. Rather, our calculation of the complexity shows that for prior $\mathrm{B}$, which gives the increased range in $r$, the data are simply sensitive to more of the parameter volume. Thus we consider the mean likelihood to be a more meaningful quantity here. In particular information on the parameters $r$ and $H_{\text {Inf }}$ under prior $\mathrm{B}$ is primarily gained on the upper bound.

As a result we emphasize that the mean likelihood for the parameters we considered gives an indication of neither a nonzero scale of inflation nor a nonzero tensorto-scalar ratio. Nevertheless, the fact that one plausible parametrization of the data increases the posterior probability of these quantities to be nonzero, suggests that from a Bayesian point of view the motivation for probing for tensor modes may be slightly enhanced as a result of our analysis.

\section{Acknowledgements}

WV acknowledges the hospitality of Case Western Reserve University, where this work was begun during a very pleasant stay. WV is supported by the EU 6th Framework Marie Curie Research and Training network "UniverseNet" (MRTN-CT-2006-035863). Numerical simulations were performed on the MUST cluster at LAPP (CNRS \& Université de Savoie). LMK is supported by grants from the US DOE and NASA, and acknowledges the hospitality of the Institute for Theoretical Physics at University of Zurich, where this work was completed. It is a pleasure to thank Andrew Liddle, David Parkinson, 
Hiranya Peiris and Roberto Trotta for helpful comments and discussions. We also thank Pascal Vaudrevange for informing us of his related work in this area.
[1] A. A. Starobinsky, Phys. Lett. B91, 99 (1980).

[2] A. H. Guth, Phys. Rev. D23, 347 (1981).

[3] K. Sato, Mon. Not. Roy. Astron. Soc. 195, 467 (1981).

[4] A. D. Linde, Phys. Lett. B108, 389 (1982).

[5] A. Albrecht and P. J. Steinhardt, Phys. Rev. Lett. 48, 1220 (1982).

[6] A. D. Linde, Phys. Lett. B129, 177 (1983).

[7] A. A. Starobinsky, JETP Lett. 30, 682 (1979).

[8] V. F. Mukhanov and G. V. Chibisov, JETP Lett. 33, 532 (1981).

[9] S. W. Hawking, Phys. Lett. B115, 295 (1982).

[10] A. A. Starobinsky, Phys. Lett. B117, 175 (1982).

[11] A. H. Guth and S. Y. Pi, Phys. Rev. Lett. 49, 1110 (1982).

[12] J. M. Bardeen, P. J. Steinhardt, and M. S. Turner, Phys. Rev. D28, 679 (1983).

[13] L. F. Abbott and M. B. Wise, Nucl. Phys. B244, 541 (1984).

[14] P. J. Steinhardt and M. S. Turner, Phys. Rev. D29, 2162 (1984).

[15] D. S. Salopek and J. R. Bond, Phys. Rev. D42, 3936 (1990).

[16] A. R. Liddle, P. Parsons, and J. D. Barrow, Phys. Rev. D50, 7222 (1994), astro-ph/9408015.

[17] L. Verde, L.-M. Wang, A. Heavens, and M. Kamionkowski, Mon. Not. Roy. Astron. Soc. 313, L141 (2000), astro-ph/9906301.

[18] M. Kamionkowski, A. Kosowsky, and A. Stebbins, Phys. Rev. Lett. 78, 2058 (1997), astro-ph/9609132.

[19] U. Seljak and M. Zaldarriaga, Phys. Rev. Lett. 78, 2054 (1997), astro-ph/9609169.

[20] M. J. Mortonson and W. Hu, Phys. Rev. D77, 043506 (2008), arXiv:0710.4162 [astro-ph].

[21] P. Martineau and R. Brandenberger (2007), arXiv:0709.2671 [astro-ph].

[22] F. Bernardeau, Astron. Astrophys. 338, 767 (1998), astro-ph/9802243.

[23] M. Zaldarriaga and U. Seljak, Phys. Rev. D59, 123507 (1999), astro-ph/9810257.

[24] A. de Oliveira-Costa et al., Phys. Rev. D68, 083003 (2003), astro-ph/0212419.

[25] M. Amarie, C. Hirata, and U. Seljak, Phys. Rev. D72, 123006 (2005), astro-ph/0508293.

[26] L. M. Krauss, Phys. Lett. B284, 229 (1992).

[27] K. Jones-Smith, L. M. Krauss, and H. Mathur, Phys. Rev. Lett. 100, 131302 (2008), arXiv:0712.0778 [astro$\mathrm{ph}]$.

[28] J. Tauber et al. (Planck) (2006), astro-ph/0604069.

[29] P. Oxley et al., Proc. SPIE Int. Soc. Opt. Eng. 5543, 320 (2004), astro-ph/0501111.

[30] K. W. Yoon et al. (2006), astro-ph/0606278.

[31] J. Bock et al. (2006), astro-ph/0604101.

[32] B. Maffei et al., Proceedings of the workshop on Dome C in Toulouse, EAS Publications Series 14, 251 (2005).

[33] C. R. Lawrence et al., Millimeter and Submillimeter Detectors for Astronomy II, Proceedings of the SPIE 5498, 220 (2004).
[34] C. J. MacTavish et al. (2007), arXiv:0710.0375 [astro-ph].

[35] J. E. Ruhl et al. (The SPT) (2004), astro-ph/0411122.

[36] A. Kogut et al., New Astron. Rev. 50, 1009 (2006), astro$\mathrm{ph} / 0609546$.

[37] G. Polenta et al., New Astronomy Review 51, 256 (2007).

[38] M. S. Turner, Phys. Rev. D48, 5539 (1993), astro$\mathrm{ph} / 9307035$.

[39] E. J. Copeland, E. W. Kolb, A. R. Liddle, and J. E. Lidsey, Phys. Rev. D48, 2529 (1993), hep-ph/9303288.

[40] E. J. Copeland, E. W. Kolb, A. R. Liddle, and J. E. Lidsey, Phys. Rev. Lett. 71, 219 (1993), hep-ph/9304228.

[41] D. H. Lyth, Phys. Rev. Lett. 78, 1861 (1997), hep$\mathrm{ph} / 9606387$.

[42] H. Peiris and R. Easther, JCAP 0607, 002 (2006), astroph/0603587.

[43] R. Easther and H. Peiris, JCAP 0609, 010 (2006), astro$\mathrm{ph} / 0604214$.

[44] H. Peiris and R. Easther, JCAP 0610, 017 (2006), astro$\mathrm{ph} / 0609003$.

[45] J. Lesgourgues and W. Valkenburg, Phys. Rev. D75, 123519 (2007), astro-ph/0703625.

[46] J. Lesgourgues, A. A. Starobinsky, and W. Valkenburg, JCAP 0801, 010 (2008), arXiv:0710.1630 [astro-ph].

[47] J. Hamann, J. Lesgourgues, and W. Valkenburg, JCAP 0804, 016 (2008), 0802.0505.

[48] C. Destri, H. J. de Vega, and N. G. Sanchez, Phys. Rev. D77, 043509 (2008), astro-ph/0703417.

[49] D. Parkinson, P. Mukherjee, and A. R. Liddle, Phys. Rev. D73, 123523 (2006), astro-ph/0605003.

[50] J. R. Bond, C. R. Contaldi, Z. Huang, L. Kofman, and P. M. Vaudrevange, in preparation.

[51] P. M. Vaudrevange, Ph.D. thesis, University of Toronto (2007), URL http://www.cita.utoronto.ca/ pascal/ pascal_thesis.pdf.

[52] R. Trotta (2008), 0803.4089.

[53] M. Bucher, J. Dunkley, P. G. Ferreira, K. Moodley, and C. Skordis, Phys. Rev. Lett. 93, 081301 (2004), astroph/0401417.

[54] M. Beltran, J. Garcia-Bellido, J. Lesgourgues, A. R. Liddle, and A. Slosar, Phys. Rev. D71, 063532 (2005), astro$\mathrm{ph} / 0501477$.

[55] M. Beltran, J. Garcia-Bellido, J. Lesgourgues, and M. Viel, Phys. Rev. D72, 103515 (2005), astro$\mathrm{ph} / 0509209$.

[56] J. Dunkley et al. (WMAP) (2008), arXiv:0803.0586 [astro-ph].

[57] M. Tegmark et al., Phys. Rev. D74, 123507 (2006), astroph/0608632.

[58] A. Lewis and S. Bridle, Phys. Rev. D66, 103511 (2002), astro-ph/0205436.

[59] D. J. Spiegelhalter, N. G. Best, B. P. Carlin, and A. van der Linde, Journal of the Royal Statistical Society: Series B (Statistical Methodology) 64, $583 \quad$ (2002), http://www.blackwellsynergy.com/doi/pdf/10.1111/1467-9868.00353, URL http://www.blackwell-synergy.com/doi/abs/10. 1111/1467-9868.00353 
[60] M. Kunz, R. Trotta, and D. Parkinson, Phys. Rev. D74, 023503 (2006), astro-ph/0602378.

[61] P. Mukherjee, D. Parkinson, and A. R. Liddle, Astro-

phys. J. 638, L51 (2006), astro-ph/0508461. 